\title{
Spatial Correlation Analysis of 2013 Per capita GDP in the Area of Beijing, Tianjin and Hebei
}

\author{
Renhao Jin, Tao Liu, Fang Yan, Jie Zhu \\ School of Information, Beijing Wuzi University, Beijing, China
}

Email address:

Renhao.jin@outlook.com (Renhao Jin)

\section{To cite this article:}

Renhao Jin, Tao Liu, Fang Yan, Jie Zhu. Spatial Correlation Analysis of 2013 Per capita GDP in the Area of Beijing, Tianjin and Hebei. American Journal of Theoretical and Applied Statistics. Vol. 4, No. 4, 2015, pp. 312-316. doi: 10.11648/j.ajtas.20150404.22

\begin{abstract}
This paper is based on the Moran's I coefficient and Geary's c coefficient, and with the support of SAS statistical analysis software, using the spatial analysis of Beijing-Tianjin-Hebei's per capita GDP and Geographical coordinates together. The research results show that the Moran's I coefficient is 0.098 , Geary's c coefficient is 0.868 , which is showing that there is a positive correlation between Beijing-Tianjin- Hebei region's city economy. But the degree of correlation is low, which is showing that Beijing-Tianj-Hebei collaborative development is still in the initial stage, and regional economic integration has not fully realized.
\end{abstract}

Keywords: Regional Economic Integration, Collaborative Development, Spatial Analysis

\section{Introduction}

There are 28 provinces and 4 municipalities directly under the Central Government in China mainland. Beijing and Tianjin are the two of the four municipalities, and Beijing is also the capital of China. Beijing also is the China capital of politics, economics, and culture, and it offer a lot of opportunities for the People. Beijing has a population of more than 21.15 million, which causes a lot of traffic, pollution and accommodation problems. However, things are a little different in Hebei Provinces, as it is ten times larger than Beijing but its population is only two times more than Beijing. Based on these problems, the central China government is promote a strategy of Regional economic integration on Beijing, Tianjin and Hebei. This paper is to investigate the status of the process of this economic integration.

The 2013 Per capita GDP data of this area and spatial autocorrelation analysis are used to measure the status of economic integration. The GDP is a comprehensive index, which can represent a lot of economic status. Considering the area of Hebei province is relative larger, Per capita GDP is used for analysis. Spatial autocorrelation is the correlation among values of a single variable strictly attributable to their relatively close locational positions. Tobler's first law of geography encapsulates this situation: "everything is related to everything else, but near things are more related than distant things." This paper use Moran's I coefficients and Geary's C coefficients to measure the Spatial autocorrelation in the Per capita GDP data for the study area. The definition of these two coefficients are explained below.

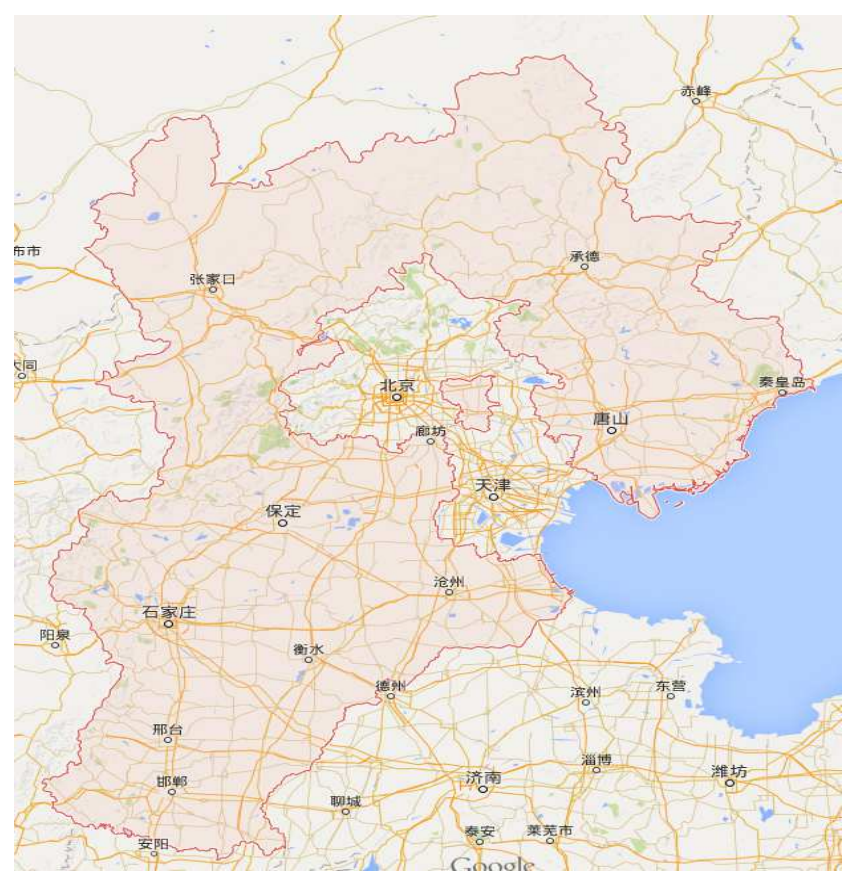

Figure 1. The map of Beijing, Tianjin, and Hebei from Google map. The dash area is Hebei Province, and the area included by Hebei and Sea is Beijing and Tianjin, which have same area size. 


\section{Data Description}

The data used in this paper are collected from the website of sohu.com. The county data of Beijing and Tianjin, and city data of Hebei Province are used, which contains per capita
GDP and longitude and latitude of the local government site. The whole data are listed in Table 1. As shown in the table, there are 16 counties in Beijing and Tianjin respectively, and 11 cites in Hebei Provinces. The distribution of per capita GDP values are displayed in the boxplot of Figure 2.

Table 1. The 2013 per capita GDP data of Beijing, Tianjin and Hebei with the longitude and latitude data.

\begin{tabular}{|c|c|c|c|c|}
\hline Area & City/county & 2013 per capita GDP (China Yuan) & Longitude & Latitude \\
\hline \multirow{16}{*}{ Beijing } & Xicheng & 214888.72 & 116.372454 & 39.918191 \\
\hline & Dongcheng & 172167.22 & 116.42276 & 39.934769 \\
\hline & Shunyi & 135350.97 & 116.661085 & 40.136507 \\
\hline & Haidian & 107130.87 & 116.309969 & 39.992281 \\
\hline & Chaoyang & 102056.76 & 116.44976 & 39.92656 \\
\hline & Daxing & 89256.80 & 116.348055 & 39.732472 \\
\hline & Shijinshan & 56677.02 & 116.229563 & 39.911342 \\
\hline & Huairou & 52460.73 & 116.638156 & 40.322312 \\
\hline & Fangshan & 48514.85 & 116.149663 & 39.754185 \\
\hline & Fengtai & 44576.20 & 116.292652 & 39.864803 \\
\hline & Miyun & 41239.50 & 116.849711 & 40.382106 \\
\hline & Mengtougou & 40990.10 & 116.10875 & 39.946234 \\
\hline & Pinggu & 39928.91 & 117.12759 & 40.146966 \\
\hline & Tongzhou & 37858.22 & 116.663214 & 39.91623 \\
\hline & Changping & 29115.93 & 116.237897 & 40.226372 \\
\hline & Yanqing & 29082.28 & 115.981706 & 40.462339 \\
\hline \multirow{15}{*}{ Tianjin } & Binhai & 304240.95 & 117.71713 & 39.009822 \\
\hline & Heping & 202227.43 & 117.221184 & 39.123191 \\
\hline & Dongli & 106618.20 & 117.320777 & 39.092262 \\
\hline & Xiqing & 99185.50 & 117.015262 & 39.147496 \\
\hline & Beichen & 97188.21 & 117.141985 & 39.23004 \\
\hline & Ninghe & 92960.29 & 117.831069 & 39.336646 \\
\hline & Jinnan & 85199.10 & 117.363487 & 38.943845 \\
\hline & Wuqing & 75002.37 & 117.050707 & 39.389024 \\
\hline & Hexi & 70411.77 & 117.229775 & 39.115759 \\
\hline & Jinghai & 66853.93 & 116.980747 & 38.953441 \\
\hline & Baodi & 55797.02 & 117.316431 & 39.723239 \\
\hline & Nankai & 52679.38 & 117.156626 & 39.144152 \\
\hline & $\mathrm{Ji}$ & 45804.40 & 117.414869 & 40.051473 \\
\hline & Hebei & 44547.40 & 117.203344 & 39.153791 \\
\hline & Hedong & 33369.21 & 117.258675 & 39.135023 \\
\hline \multirow{12}{*}{ Hebei } & Hongqiao & 28404.05 & 117.157216 & 39.173205 \\
\hline & Tangshan & 79365.20 & 118.186678 & 39.636637 \\
\hline & Shijiazhuang & 46828.20 & 114.521409 & 38.048234 \\
\hline & Langfang & 44854.57 & 116.69041 & 39.544007 \\
\hline & Cangzhou & 40571.77 & 116.845322 & 38.310336 \\
\hline & Qinhuangdao & 38681.49 & 119.608614 & 39.941588 \\
\hline & Chengde & 34107.95 & 117.969493 & 40.959115 \\
\hline & Handan & 30827.71 & 114.545866 & 36.631195 \\
\hline & Zhangjiakou & 29974.05 & 114.892592 & 40.774341 \\
\hline & Hengshui & 25090.42 & 115.676782 & 37.745031 \\
\hline & Baoding & 23609.42 & 115.47105 & 38.880045 \\
\hline & Xingtai & 22321.45 & 114.511441 & 37.076789 \\
\hline
\end{tabular}


Boxplot of 2013 per capita GDP in Beijing, Tianjin and Hebei

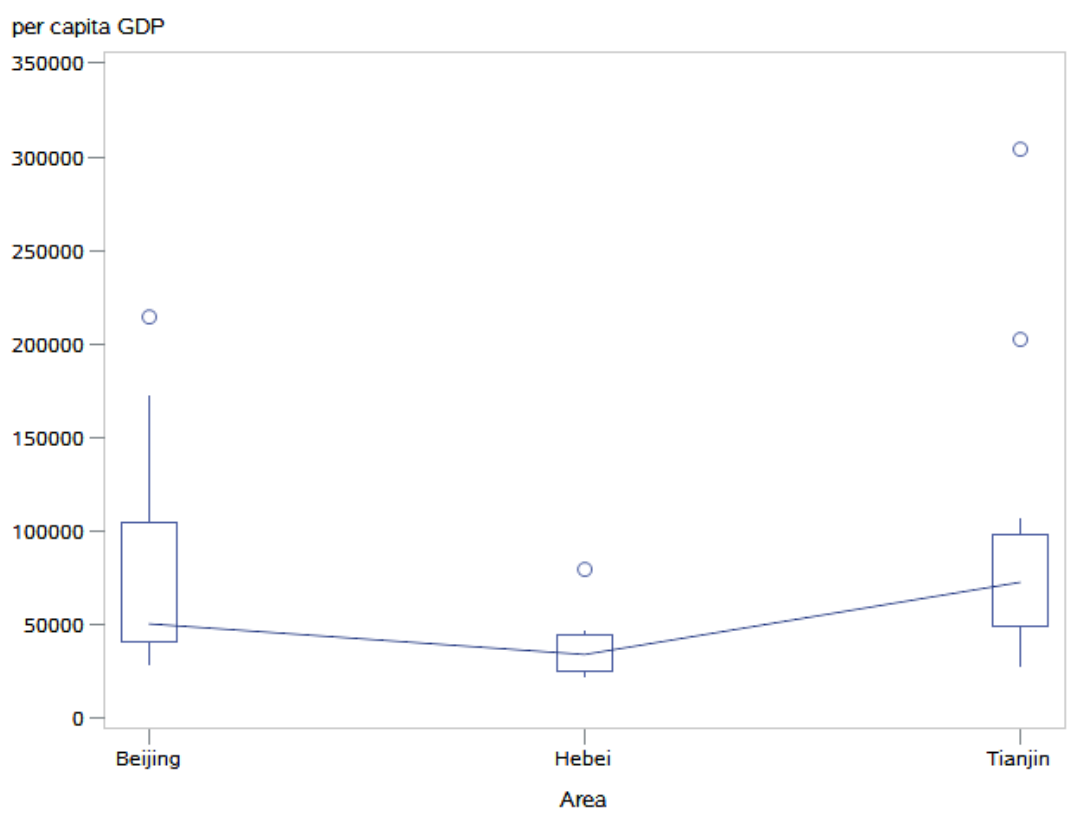

Figure 2. The boxplot of 2013 per capita GDP in Beijing, Tianjin and Hebei.

From the Figure 2, the median per capita GDP of the counties in Tianjin are the highest, and the counterpart of Beijing is in the second place. At the same time, the fluctuations in Tianjin's GDP data are larger than the remaining two areas. In the Figure 3, spatial distribution of 2013 per capita GDP data of Beijing, Tianjin and Hebei are displayed. Comparing Figure 1 and Figure 3, it can be found that the part with relative larger circles $(39<\mathrm{x}<40.5$ and $116<y<118)$ are Beijing and Tianjin, and from the spatial distribution the spatial autocorrelations can be seen in the GDP data. The counties around and in Beijing and Tianjin area are tended to large values, while the counties, far away from this area are tended to be small values. To make an accurate inference, Moran's I coefficient and Geary's c coefficient are introduced in the next section.

\section{Spatial distribution of $\mathbf{2 0 1 3}$ per capita GDP data of Beijing, Tianjin and Hebei}

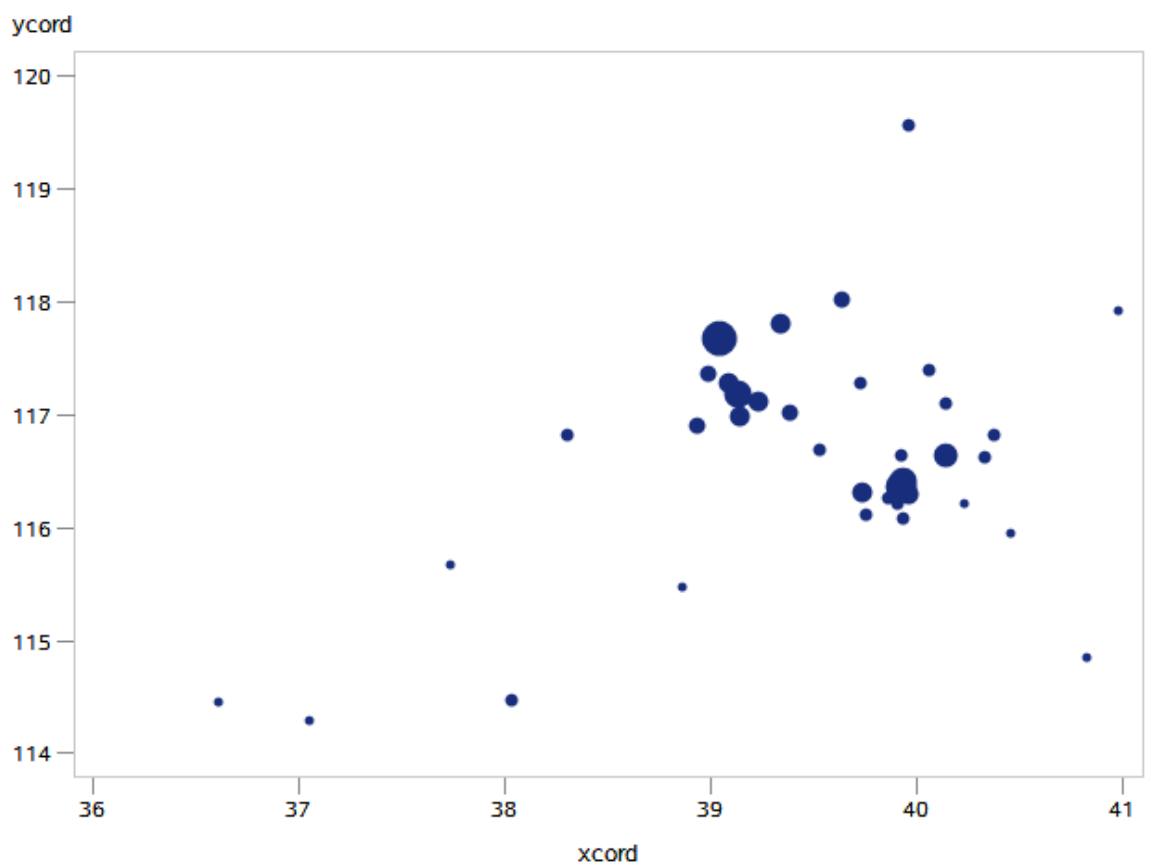

Figure 3. The spatial distribution of 2013 per capita GDP data of Beijing, Tianjin and Hebei. The size of the circles represents the scale of the per capita GDP values in such counties. 


\section{Moran's I and Geary's c}

The Moran's I coefficient are defined as

$$
I=\frac{n}{\sum_{i=1}^{n} \sum_{j=1}^{n} w_{i j}} \frac{\sum_{i=1}^{n} \sum_{j=1}^{n} w_{i j}\left(x_{i}-\frac{\sum_{i} x_{i}}{n}\right)\left(x-\frac{\sum_{j=1}^{n} x_{j}}{n}\right)}{\sum_{i=1}^{n}\left(x_{i}-\frac{\sum_{i=1}^{n} x_{i}}{n}\right)^{2}}
$$

and the Geary's c coefficient are calculated as

$$
C=\frac{(n-1) \sum_{i=1}^{n} \sum_{j=1}^{n} w_{i j}\left(x_{i}-x_{j}\right)^{2}}{2 \sum_{i=1}^{n} \sum_{j=1}^{n} w_{i j} \sum_{i=1}^{n}\left(x_{i}-\bar{x}\right)^{2}} .
$$

In statistics, Moran's I is a measure of spatial autocorrelation developed by Patrick A.P. Moran. Like autocorrelation, spatial autocorrelation means that adjacent observations of the same phenomenon are correlated. However, autocorrelation is about proximity in time. Spatial autocorrelation is about proximity in (two-dimensional) space. Spatial autocorrelation is more complex than autocorrelation because the correlation is two-dimensional and bi-directional. Negative (positive) values indicate negative (positive) spatial autocorrelation. Values range from -1 (indicating perfect dispersion) to +1 (perfect correlation). A zero values indicates a random spatial pattern. For statistical hypothesis testing, Moran's I values can be transformed to Z-scores in which values greater than 1.96 or smaller than -1.96 indicate spatial autocorrelation that is significant at the $5 \%$ level. The Z-scores transformation can be easily written as

$$
Z=\frac{I-E(I)}{\sqrt{\operatorname{VAR}(I)}} .
$$

Geary's C is also a measure of spatial autocorrelation or an attempt to determine if adjacent observations of the same phenomenon are correlated. Geary's C is inversely related to Moran's I, but it is not identical. Moran's I is a measure of global spatial autocorrelation, while Geary's $\mathrm{C}$ is more sensitive to local spatial autocorrelation. Geary's $\mathrm{C}$ is also known as Geary's contiguity ratio or simply Geary's ratio. The value of Geary's C lies between 0 and 2. 1 means no spatial autocorrelation. Values lower than 1 demonstrate increasing positive spatial autocorrelation, whilst values higher than 1 illustrate increasing negative spatial autocorrelation.

The Moran's I coefficient and Geary's c coefficient are calculated and shown in table 2. It can be seen that the 2013 per capita GDP data in the study area are displayed positive autocorrelation both from Moran's I coefficient and Geary's c coefficient, which means the per capita GDP data in the nearby counties or cities are tended to similar. The $p$-value of the spatial autocorrelation test are 0.3395 and 0.5725 for Moran's I coefficient and Geary's c coefficients respectively. However, at the same time it is also noticed that only week positive autocorrelation are found in the data, as the Moran's I coefficient are close to 0 , and Geary's c coefficient are close to 1.

Table 2. The Moran's I coefficient and Geary's c coefficient are computed from the 2013 per capita GDP in counties or cities in Beijing, Tianjin and Hebei.

\begin{tabular}{llll}
\hline \multicolumn{4}{l}{ Spatial autocorrelation coefficients } \\
\hline Assumption & Coefficient & Observed & $\operatorname{Pr}>|\mathbf{Z}|$ \\
\hline Randomization & Moran's I & 0.098 & 0.3395 \\
Randomization & Geary's C & 0.868 & 0.5725 \\
\hline
\end{tabular}

\section{Conclusion}

This paper use Moran's I coefficients and Geary's C coefficients to measure the Spatial autocorrelation in the Per capita GDP data for the study area to investigate the status of the process of the region economic integration. Based on the results from Figure 2 and Table 2, it can conclude that only week positive autocorrelation are found in this region, and the Per capita GDP value is high in Beijing and Tianjin area. It can be predicted that the center of region economic integration should be around in Beijing and Tianjin, hopefully it can gradually promote the whole area economics. From the Moran's I coefficient and Geary's c coefficient, it can find that the extent of region economic integration is still low and need more investments to increase the integration extent. Meanwhile, to solve the traffic and population problem in Beijing, the government should make more people and companies move to Hebei area by economic inspiring and better polices.

\section{Acknowledgements}

This paper is funded by the project of National Natural Science Fund, Logistics distribution of artificial order picking random process model analysis and research (Project number: 71371033); and funded by intelligent logistics system Beijing Key Laboratory (No.BZ0211); and funded by scientific-research bases---Science \& Technology Innovation Platform---Modern logistics information and control technology research (Project number: PXM2015_014214_000001); University Cultivation Fund Project of 2014-Research on Congestion Model and algorithm of picking system in distribution center (0541502703).

\section{References}

[1] Anselin, L. (1995). Local indicators of spatial association LISA. Geographical Analysis 27, 93--115. 
[2] Besag, J. (1974). Spatial interaction and the statistical analysis of lattice systems. Journal of the Royal Statistical Society B 36, $192--225$.

[3] Cressie, N., \& Hawkins, D.M. (1980). Robust estimation of variogram: I. Mathematical Geology 12: 115-125.

[4] Diggle, P.J., \& Ribeiro, P.J. (2007). Model-based geostatistics, New York: Springer.

[5] Glatzer, E., \& Müller, W.G. (2004). Residuals diagnostics for variogram fitting. Computers and Geosciences $30: 859-866$.

[6] Getis, A. and Ord, J. K. (1992). The analysis of spatial association by use of distance statistics. Geographical Analysis 24, 189--206.

[7] Griffith, D. (1987). Spatial Autocorrelation: A Primer. Washington, DC: Association of American Geographers Resource Publication.
[8] Griffith, D. (1992). What is spatial autocorrelation? Reflections on the past 25 years of spatial statistics. l'Espace Ge'ographique 21, 265--280.

[9] Griffith, D. (1996). Spatial autocorrelation and eigenfunctions of the geographic weights matrix accompanying geo-referenced data. The Canadian Geographer 40, 351--367.

[10] Mardia, K. and Marshall, R. (1984). Maximum likelihood estimation of models for residual covariance in spatial regression. Biometrika 71, 135--146.

[11] Richardson, S. and He' mon, D. (1981). On the variance of the sample correlation between two independent lattice processes. Journal of Applied Probability 18, 943--948.

[12] Tiefelsdorf, M. and Boots, B. (1995). The exact distribution of Moran's I. Environment and Planning A 27, 985--999. 